\title{
A stellar population synthesis model for the study of ultraviolet star counts of the Galaxy
}

\author{
Ananta C. Pradhan ${ }^{1}$, Devendra K. Ojha ${ }^{1}$, Annie C. Robin ${ }^{2}$, Swarna K. Ghosh ${ }^{1,3}$, and John J. Vickers ${ }^{\star, 4}$ \\ 1 Tata Institute of Fundamental Research, Homi Bhabha Road, 400005 Mumbai, India \\ e-mail: acp.phy@gmail.com \\ 2 Institut Utinam, CNRS UMR 6213, OSU THETA, Université de Franche-Comté, 41bis avenue de l'Observatoire 25000 Besançon, \\ France \\ 3 National Centre for Radio Astrophysics, Tata Institute of Fundamental Research, 411007 Pune, India \\ 4 Astronomisches Rechen-Institut, Zentrum für Astronomie der Universität Heidelberg, Mönchhofstr, 12-14, 69120 Heidelberg, \\ Germany
}

Received 23 April 2013 / Accepted 11 March 2014

\section{ABSTRACT}

\begin{abstract}
Context. Galaxy Evolution Explorer (GALEX), the first all sky imaging ultraviolet (UV) satellite, has imaged a large part of the sky providing an excellent opportunity for studying UV star counts. Combining photometry from the different wavelengths in the infrared (from Wide-field Infrared Survey (WISE) and Two Micron All Sky Survey (2MASS)) to UV allows us to extract a real star catalogue from the GALEX source catalogue.

Aims. The aim of our study is to investigate in detail the observed UV star counts obtained by GALEX vis-à-vis the model simulated catalogues produced by the Besançon model of stellar population synthesis in various Galactic directions, and to explore the potential for studying the structure of our Galaxy from images in multiple near-UV (NUV) and far-UV (FUV) filters of the forthcoming Ultraviolet Imaging Telescope (UVIT) to be flown onboard Astrosat.

Methods. We have upgraded the Besançon model of stellar population synthesis to include the UV bands of GALEX and UVIT. Depending on the availability of contiguous GALEX, Sloan Digital Sky Survey (SDSS), WISE, and 2MASS overlapping regions, we have chosen a set of 19 GALEX fields which spread over a range of Galactic directions. We selected a sample of objects from the GALEX database using the CASjobs interface and then cross-matched them with the WISE+2MASS and SDSS catalogues. The UV stars in the GALEX catalogue are identified by choosing a suitable infrared (IR) colour, $J-W 1$ (W1 is a WISE band at $3.4 \mu \mathrm{m}$ ), which corresponds to a temperature range from $1650 \mathrm{~K}$ to $65000 \mathrm{~K}$. The IR colour cut method, which is used for the first time for separation of stars, is discussed in comparison with the GALEX+SDSS star counts method.

Results. We present the results of the UV star counts analysis carried out using the data from GALEX. We find that the Besançon model simulations represent the observed star counts of both the GALEX All-sky Imaging Survey and Medium Imaging Survey well within the error bars in various Galactic directions. Based on the analysis of the model $F U V-N U V$ colour, we separated out white dwarfs of the disc and blue horizontal branch stars of the halo from the observed sample by selecting a suitable $F U V-N U V$ colour. Conclusions. The Besançon model is now ready for further comparisons in the UV domain and will be used for prospective studies for the UVIT instrument to be flown onboard Astrosat.
\end{abstract}

Key words. stars: general - ultraviolet: stars - Galaxy: disk - Galaxy: stellar content - Galaxy: halo

\section{Introduction}

The Milky Way is the best studied Galaxy in the universe; its structure and evolution have been studied using a variety of techniques. In the early 20th century, Kapteyn (1922) first studied the geometrical structure of the Galaxy using the star counts method whereby he counted stars on the photographic plates in selected areas of the sky. Since then the star counts method has been used as one of the preferred methods to constrain the structural parameters of the Galaxy effectively. Several reviews have discussed the connection of star counts to the Galactic structure (Bahcall 1986; Freeman 1987; Gilmore et al. 1988; Majewski 1993; Helmi 2008; Ivezić et al. 2012). The advent of instruments with better resolution and greater sensitivity have enabled us to obtain photometric observations covering large parts of the sky in several wavelength bands. The population synthesis models of the Milky Way are well supported by these observations

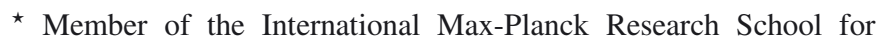
Astronomy \& Cosmic Physics at the University of Heidelberg. in predicting the different structural parameters of the Galaxy, such as stellar densities, scale length, scale height, etc. Among the models developed to understand the Galactic structure using star counting methods, one can cite: Bahcall \& Soneira (1980), Gilmore \& Reid (1983), Robin \& Crézé (1986), Robin et al. (2003), Girardi et al. (2005) and Jurić et al. (2008). However, the above Galaxy models are predominantly based on the visible and infrared (IR) photometric surveys. Very few attempts had been made to study the star counts in ultraviolet (UV) prior to Galaxy Evolution Explorer (GALEX) because of lack of availability of UV photometric surveys (Brosch 1991; Cohen et al. 1994). The advent of GALEX, which provided a wide sky coverage in UV, now allows for a new analysis of the UV sky (Xu et al. 2005; Bianchi et al. 2011a,b, 2014, among others). An attempt has also been made to predict the star counts in the X-ray band (Guillout et al. 1996) by extending the Besançon model of stellar population synthesis (Robin \& Crézé 1986) to the ROSAT position sensitive proportional counter energy bands. 

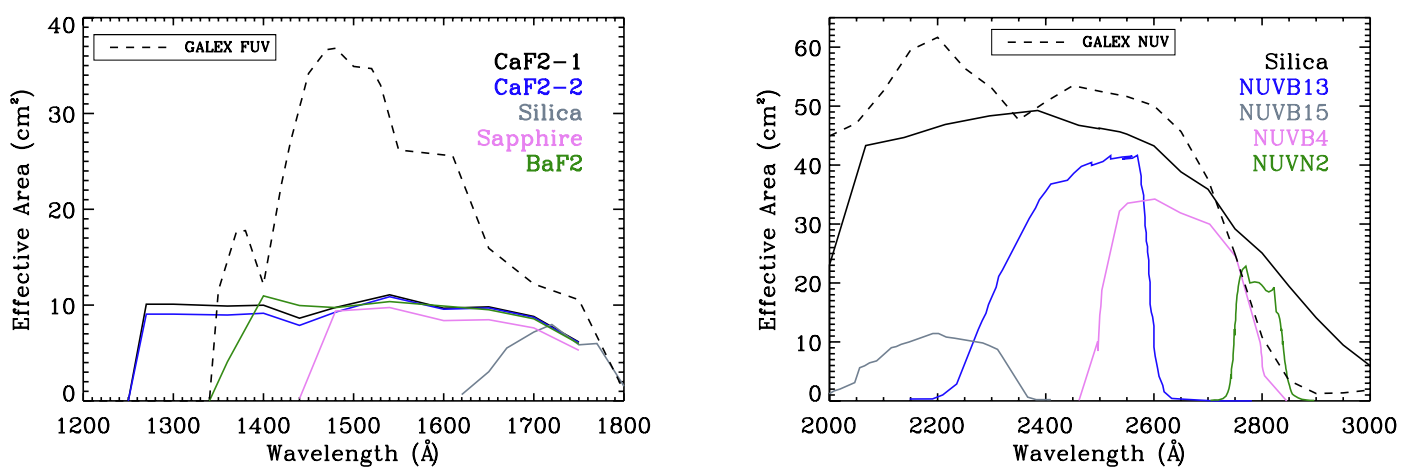

Fig. 1. Effective area versus wavelength plots for the GALEX FUV and NUV bands are shown in relation to the FUV and NUV filters of UVIT/Astrosat for imaging mode. The left panel shows the five FUV filters of UVIT/Astrosat in different colours along with the GALEX FUV filter (dashed line) and the right panel shows the same for the NUV filters.

Indeed, the UV surveys, among others, could help in tracing the spiral structures which mainly contain very young stars. The UV surveys also help in constraining the shape of the initial mass function (IMF) towards the high-mass star end as well as elucidating the recent star formation history. Moreover, they also trace very blue populations such as white dwarfs (WDs) and blue horizontal branch stars (BHBs) deep in the halo population, which in turn trace the streams and relics of ancient accretion in the Milky Way halo. The GALEX has covered a large part of the sky, which provides an opportunity to explore and characterise these hot sources in the far-UV (FUV: 1344$1786 \AA, \lambda_{\text {eff }}=1538.6 \AA$ ) and near-UV (NUV: 1771-2831 $\AA$, $\lambda_{\text {eff }}=2315.7 \AA$ ) wavebands with better resolution and greater sensitivity than the previous surveys. A vivid description of the source selection, FUV and NUV magnitude error cuts, and the statistical analysis of the GALEX catalogue is provided by Bianchi et al. (2007), Bianchi (2009), and Bianchi et al. (2011a, 2014). Detection of WDs and BHBs is one of the main achievements of GALEX as these sources are elusive in the other wavelength bands of the electromagnetic spectrum due to their high temperature. The population of WDs and BHBs is integral to the study of stellar evolution and structure of the Milky Way as they belong to different stellar populations of the Galaxy.

We have upgraded the Besançon model of stellar population synthesis to include the UV bands of GALEX and the upcoming Ultraviolet Imaging Telescope (UVIT ${ }^{1}$ ) (which will be flown onboard Astrosat) to predict star counts in different parts of the sky (Todmal et al. 2010). The UVIT will image the sky in the FUV (1300-1800 ̊) and NUV (2000-3000 ̊) channels, each having five filters, at a high resolution of $1.8^{\prime \prime}$ (Postma et al. 2011; Kumar et al. 2012a,b). Better positional accuracy of UVIT as compared to GALEX will enable more reliable cross correlation with other catalogues, which will be of great utility in inferring the Galactic structure using the star counts technique. The transmission curves (effective area versus wavelength) for the FUV and NUV bands of GALEX with each of the five FUV (left panel) and NUV (right panel) filters of the upcoming UVIT/Astrosat are shown in Fig. 1. We have included the effective area curves of both the GALEX and all the UVIT/Astrosat bands in the model to simulate the UV star counts in these bands. Apart from the GALEX bands, we will discuss the model simulated star counts of the BaF2 (FUV: 1370-1750 $\mathrm{A}, \lambda_{\text {eff }}=1504 \AA$ ) and NUVB4 (NUV: $2505-2780 \AA$, $\lambda_{\mathrm{eff}}=2612 \AA$ ) bands of UVIT/Astrosat. The expected sensitivity limits $(5 \sigma)$ in $\mathrm{AB}$ magnitude system in the UVIT BaF2 (FUV)

\footnotetext{
1 http://www.iiap.res.in/Uvit
}

and NUVB4 (NUV) wavebands, for an exposure time of $200 \mathrm{~s}$, are 20.0 and 21.2 mag, respectively (Astrosat Handbook 2013; priv. comm.).

It is worth mentioning here that throughout the paper we have used AB system for the GALEX, UVIT, and Sloan Digital Sky Survey (SDSS) data sets, whereas the Two Micron All Sky Survey (2MASS) and Wide-field Infrared Survey (WISE) data sets are in the Johnson system (see Sect. 2).

We give details of the observations and selection of UV stars in Sect. 2. We describe the Besançon Galaxy model in Sect. 3 and discuss the comparison of the GALEX+WISE+2MASS and GALEX+SDSS star counts in Sect. 4. We present the comparison of the model with the observations in Sect. 5, and discuss the distribution of the model star counts in Sect. 6. We mention the identification of WDs and BHBs using FUV - NUV colour in Sect. 7. Finally, we summarise our conclusions in Sect. 8.

\section{Observations and cross-correlation of GALEX sources}

\subsection{GALEX data}

The GALEX was an orbiting space telescope launched in April, 2003, which was terminated in mid-February, 2012. The satellite and on-orbit performance are described in Martin et al. (2005) and Morrissey et al. (2005, 2007). It observed the sky in two UV bands, FUV, and NUV, simultaneously, with a spatial resolution of 4.2" and 5.3", respectively. The field of view is $1.25^{\circ}$ in diameter and the images are sampled with $1.5^{\prime \prime}$ pixels. The typical AB magnitude limits ( $5 \sigma$ depth) met by Allsky Imaging Survey (AIS) for an exposure time of $100 \mathrm{~s}$ and Medium Imaging Survey (MIS) for an exposure time of $1500 \mathrm{~s}$ are 19.9/20.8 (FUV/NUV) and 22.6/22.7 (FUV/NUV), respectively (Morrissey et al. 2007). The AIS has the largest sky coverage when compared to the other GALEX surveys. The GALEX observations have covered a large part of the sky $(\sim 75 \%)$, with the exception of the Galactic plane and some regions of the Magellanic Clouds due to safety concerns of the detectors. In this paper, we have used the GALEX GR6 data which is available in Multi-mission Archive at Space Telescope Science Institute $\left(\mathrm{MAST}^{2}\right)$.

2 http://galex.stsci.edu/GR6 
Table 1. Details of the GALEX fields.

\begin{tabular}{ccccccccc}
\hline \hline Field & $\begin{array}{c}\text { Longitude } \\
\text { range }(\mathrm{deg})\end{array}$ & $\begin{array}{c}\text { Latitude } \\
\text { range }(\mathrm{deg})\end{array}$ & Survey type & $\begin{array}{c}\text { Area } \\
\left(\mathrm{deg}^{2}\right)\end{array}$ & Location & Number of tiles & $\begin{array}{c}\text { NUV exposure } \\
\text { time (s) }\end{array}$ & $\begin{array}{c}\text { FUV exposure } \\
\text { time (s) }\end{array}$ \\
\hline 1 & 47.79 & -43.56 & AIS & 0.785 & GC & 1 & 175 & 175 \\
2 & 129.33 & -43.15 & AIS & 0.785 & GAC & 1 & 258 & 258 \\
3 & 47.01 & -42.65 & MIS & 0.785 & GC & 1 & 1589 & 1589 \\
4 & 146.57 & -46.51 & MIS & 0.785 & GAC & 1 & 1657 & 1657 \\
5 & $1-15$ & $50-60$ & AIS & 69.9 & GC & 89 & $64-400$ & $64-272$ \\
6 & $160-175$ & $50-60$ & AIS & 69.9 & GAC & 89 & $90-442$ & $96-220$ \\
7 & $13-29$ & $35-41$ & MIS & 22.77 & GC & 29 & $1541-2176$ & $1541-2176$ \\
8 & $154-162$ & $38-44$ & MIS & 18.85 & GAC & 24 & $1597-4457$ & $1512-3066$ \\
9 & $230-240$ & $42-50$ & AIS & 46.34 & GAR & 59 & $80-438$ & $61-231$ \\
10 & $40-50$ & $75-85$ & AIS & 14.92 & GHL & 19 & $62-292$ & $62-249$ \\
11 & $30-32$ & $21-29$ & AIS & 13.35 & GLL & 17 & $105-421$ & $105-230$ \\
12 & $0-20$ & $84-88$ & AIS & 4.71 & GP & 6 & $107-383$ & $107-271$ \\
13 & $49-51$ & $17-23$ & AIS & 7.07 & - & 9 & $130-199$ & $130-199$ \\
14 & $48-53$ & $29-33$ & AIS & 11.0 & - & 14 & $79-184$ & $79-184$ \\
15 & $46-53$ & $37-43$ & AIS & 20.42 & - & 28 & $96-384$ & $95-265$ \\
16 & $46-54$ & $46-54$ & AIS & 17.28 & - & 22 & $94-345$ & $73-226$ \\
17 & $46-54$ & $57-63$ & AIS & 11.78 & - & 15 & $152-342$ & $152-229$ \\
18 & $46-54$ & $67-73$ & AIS & 7.07 & - & 9 & $90-340$ & $90-169$ \\
19 & $44-54$ & $77-83$ & AIS & 4.71 & - & 6 & $75-259$ & $75-151$ \\
\hline
\end{tabular}

Notes. The areas of different fields are chosen depending on the availability of GALEX, WISE+2MASS, and SDSS overlapping regions.

\subsection{Selection of GALEX fields}

We have selected 19 GALEX fields for which both the detectors of GALEX were turned on. We retained sources which had a reliable NUV detection, however, FUV detections are available for $\sim 3.5 \%$ and $\sim 6.8 \%$ of the NUV detections in the selected AIS and MIS fields, respectively. The rest of the NUV sources do not have a FUV detection because their FUV fluxes are too low to be detected. We include only regions within a radius of $0.5^{\circ}$ from the centre of the tiles to eliminate edge artifacts and bad sources along the edge as well as to avoid overlapping areas and duplication of the sources. The coverage areas of the observed fields are calculated by summing up the areas of all the tiles in a field.

The fields are selected in the footprints of the GALEX, SDSS, WISE, and 2MASS surveys. The various fields are as follows:

- Four GALEX tiles were chosen at the southern Galactic latitudes: two each in AIS and MIS.

- Eight fields with large area coverage of the sky were chosen in several northern Galactic directions. The fields include: two regions towards the Galactic centre (GC) (one each in AIS and MIS), two regions towards the Galactic anti-centre (GAC) (one each in AIS and MIS), and one region each in AIS towards the Galactic anti-rotation (GAR), Galactic low latitude (GLL), Galactic high latitude (GHL), and Galactic pole (GP) directions.

- Seven fields in AIS were chosen at $10^{\circ}$ intervals of $b$ around $l \sim 50^{\circ}$ to study the latitude variation of UV star counts.

The centre coordinates, survey types, area coverages, location in the Galaxy, number of GALEX tiles, and the range of exposure times of NUV and FUV observations of each of the fields are given in Table 1.

\subsection{WISE+2MASS data}

The AIS and MIS of GALEX overlap with the 2MASS and WISE footprints. The 2MASS observations have covered the entire sky in the $J(1.24 \mu \mathrm{m}), H(1.66 \mu \mathrm{m})$, and $K_{s}(2.16 \mu \mathrm{m})$ near-IR (NIR) bands with angular resolutions of 2.9", 2.8", and 2.9", respectively (Skrutskie et al. 2006); while WISE has mapped the sky in the W1 $(3.4 \mu \mathrm{m}), \mathrm{W} 2(4.6 \mu \mathrm{m}), \mathrm{W} 3(12 \mu \mathrm{m})$, and W4 $(22 \mu \mathrm{m})$ mid-IR bands, with angular resolutions of $6.1^{\prime \prime}$, 6.4", 6.5", and 12.0", respectively (Wright et al. 2010). The $5 \sigma$ point source sensitivities of the four WISE bands are better than $0.08,0.11,1$, and $6 \mathrm{mJy}$ (equivalent to $16.6,15.6,11.3$, and 8.0 Vega magnitude) in unconfused regions on the ecliptic (Wright et al. 2010). The existing WISE+2MASS cross-matched catalogue available at Infrared Science Archive (IRSA ${ }^{3}$ ) has been used for convenience. This catalogue has been produced using a 3.0" matching radius, which was found to be adequate considering the positional accuracy and resolution.

We have made use of the Virtual Astronomical Observatory $\left(\mathrm{VAO}^{4}\right)$ for cross-matching GALEX sources with WISE+2MASS sources. The GALEX sources were uploaded into the VAO, seeking their WISE and 2MASS counterparts using a match radius of $3.0^{\prime \prime}$. We found most of the real matched sources within this radius, with a very small fraction $(<1 \%)$ having multiple matches, which were removed from the final catalogue. We also estimated the possible contamination by spurious matches (random coincidences) for the matched sources following the method of Bianchi et al. (2011a). For this purpose, we used a match radius of $6.0^{\prime \prime}$, which is equivalent to the resolution of WISE, to find the GALEX counterparts of WISE sources. The spurious matches were found to be $\sim 10 \%$ of the total matched sources, and $75 \%$ of these spurious matched sources lie beyond a distance of $3.0^{\prime \prime}$.

\subsection{SDSS data}

So far, SDSS has mapped over 35\% of the full sky in five optical photometric bands $(u, g, r, i, z)$ covering the wavelength range from 3000 to $11000 \AA$ (Aihara et al. 2011). The GALEX

\footnotetext{
3 http://irsa.ipac.caltech.edu/Missions/wise.html

4 http://vao-web.ipac.caltech.edu/applications/VAOSCC
} 

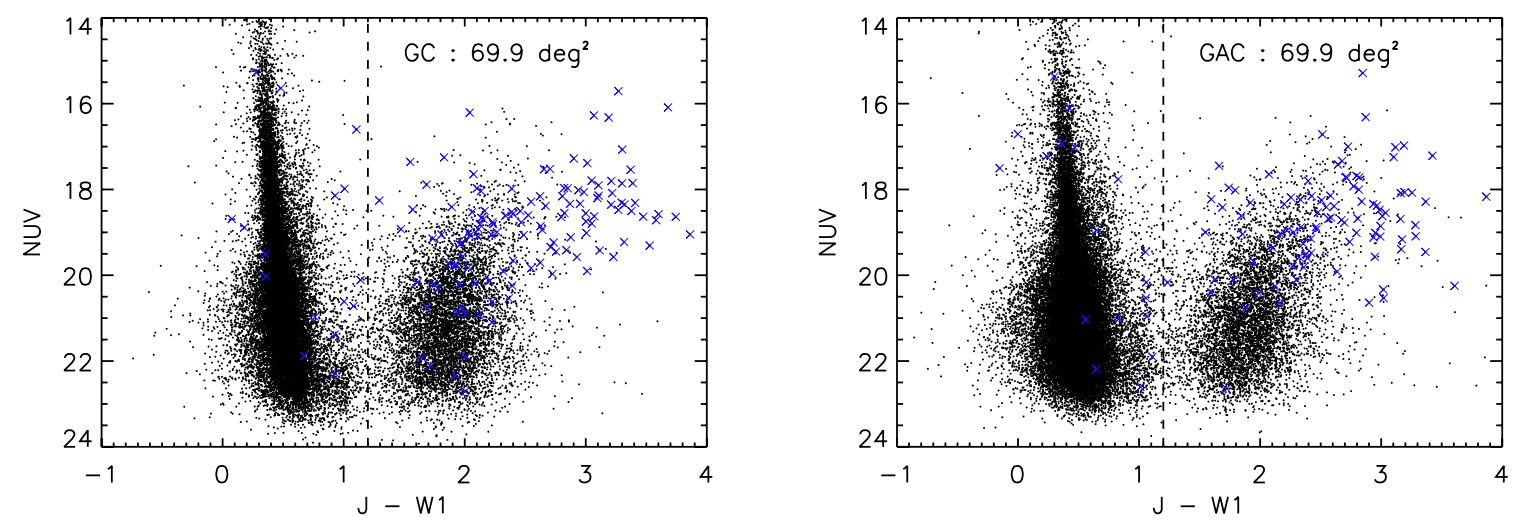

Fig. 2. $J$ (2MASS) - W1 (WISE) versus NUV CMD for the GALEX and WISE+2MASS cross-matched sources for the AIS fields towards the $\mathrm{GC}$ and the GAC. The matched sources are clearly separated in two groups indicating isolation of stars $(J-W 1<1.2)$ from the extra-galactic sources $(J-W 1>1.2)$. The vertical dashed line shows the limit that we choose for selecting the point sources $(J-W 1<1.2)$. QSOs are shown by blue crossed symbols (see the text).

GR6 has been cross-matched against SDSS DR7 and the provided cross-matched table is $x S D S S D R 7$. Several works have explained the cross-matching of the GALEX catalogue with SDSS, astrophysical source classifications and related statistical analyses (Seibert et al. 2005; Budavári et al. 2009; Bianchi et al. 2007, 2011a). We uploaded the GalexIDs of the objects into the GALEX CASjobs ${ }^{5}$ SQL (Structured Query Language) interface to determine their SDSS counterparts in a search radius of $3.0^{\prime \prime}$. We have eliminated the multiple matches $(<1 \%)$ from the GALEX+SDSS final catalogue. The estimated spurious matches in case of GALEX+SDSS are found to be $\sim 7 \%$ within $3.0^{\prime \prime}$ radius. The SDSS star/galaxy classifications have been adopted while performing the match to separate out point sources from the source list.

The SDSS classified point sources (GALEX+SDSS) include both stars and quasi-stellar objects (QSOs), out of which we selected QSOs using the SDSS colour cuts from Richards et al. (2002) and removed them from the GALEX+SDSS point sources and termed the clean sample as "GALEX+SDSS stars".

\subsection{Selection of stars from the GALEX catalogue by IR colour cut method}

Figure 2 shows $J-W 1$ versus NUV colour-magnitude diagram (CMD) of all GALEX sources that are cross-matched with WISE+2MASS sources for the regions in the directions of the GC and the GAC, each covering $69.9 \mathrm{deg}^{2}$ of the sky. The QSO candidates are selected using the SDSS colour cuts from Richards et al. (2002) and are represented by blue crossed symbols in the plot. We clearly see two groups of sources in the figure well separated by $J-W 1$ colour. The stars verified by their SDSS classification as point sources in a cross-matched sample are identified to be bluer than $J-W 1<1.2$ and the extra-galactic objects (e.g., galaxies, QSOs, etc.) are redder, with $J-W 1>1.2$. Since the contamination by SDSS-identified QSOs is estimated to be negligible in the $J-W 1<1.2$ star counts $(<0.1 \%$ of the whole sample), we have used the $J-W 1$ colour cut procedure for all the fields to separate the stars from the extragalactic objects. Henceforth in the paper, we refer to GALEX and WISE+2MASS cross-matched sources with $J-W 1<1.2$ (GALEX+WISE+2MASS) as "UV-IR stars".

\footnotetext{
5 http://galex.stsci.edu/casjobs
}

\subsection{Photometric error cuts and completeness limits}

Figure 3 shows the distribution of UV-IR stars as a function of the GALEX UV magnitudes for AIS and MIS. Stars with NUV and FUV magnitude errors less than $0.5,0.4,0.3,0.2$, and 0.15 are displayed with magenta, green, red, blue, and cyan colour lines respectively, whereas the black line represents the stars without any magnitude error cut. The typical $5 \sigma$ magnitude limits of the NUV and FUV bands for AIS and MIS (see Sect. 2.1) are shown by vertical dashed lines. As seen from the histograms, a progressive stringent error cut eliminates the fainter stars. The completeness limits need to be established according to a given magnitude error. If we consider all stars without accounting for errors, the star counts go deeper but their values are not reliable because of the uncertainty on the magnitude measurement. This is particularly true for the FUV filter where some spurious detections can occur. Finally, we retained stars with magnitude error less than 0.2 in both bands as this error cut gives magnitude limits almost similar to the typical $5 \sigma$ limits of the GALEX bands for AIS and MIS which are provided by Morrissey et al. (2007).

We have applied magnitude error cuts (similar to the one shown in Fig. 3 for UV-IR stars) in the original GALEX source catalogue that includes all the GALEX detections. We have also applied magnitude error cuts in the matched GALEX source catalogue obtained after cross-matching with the WISE+2MASS catalogue. We find a loss of GALEX sources in the matched catalogue when compared with the original GALEX source catalogue at a specific magnitude error cut. The completeness limits for the original GALEX sources for NUV and FUV magnitude error cuts of 0.2 are 20.5/21.0 mag (FUV/NUV) in AIS and 22.5/22.5 mag (FUV/NUV) in MIS. The completeness limits at the same magnitude error cuts for the matched catalogue become 20.0/20.5 mag (FUV/NUV) in AIS and 22.5/22.0 mag (FUV/NUV) in MIS, and these limits are the same for the UV-IR stars too. For a specific magnitude error cut, the FUV and NUV completeness limits of the observed sources, which are cross-matched to the surveys at longer wavelengths, become brighter than the completeness limits of the unmatched GALEX source catalogue because of the loss of faint sources in the former.

In order to examine which objects are affected by the limits of the combined surveys (GALEX+WISE+2MASS), we split the stars into two $N U V-W 1$ colour intervals: hot $(N U V-W 1<$ $5)$ and cool $(N U V-W 1>5)$ stars. We checked the completeness limit of the NUV band (AIS) for these two colour ranges. 
Ananta C. Pradhan et al.: A Model for the UV star counts of the Galaxy
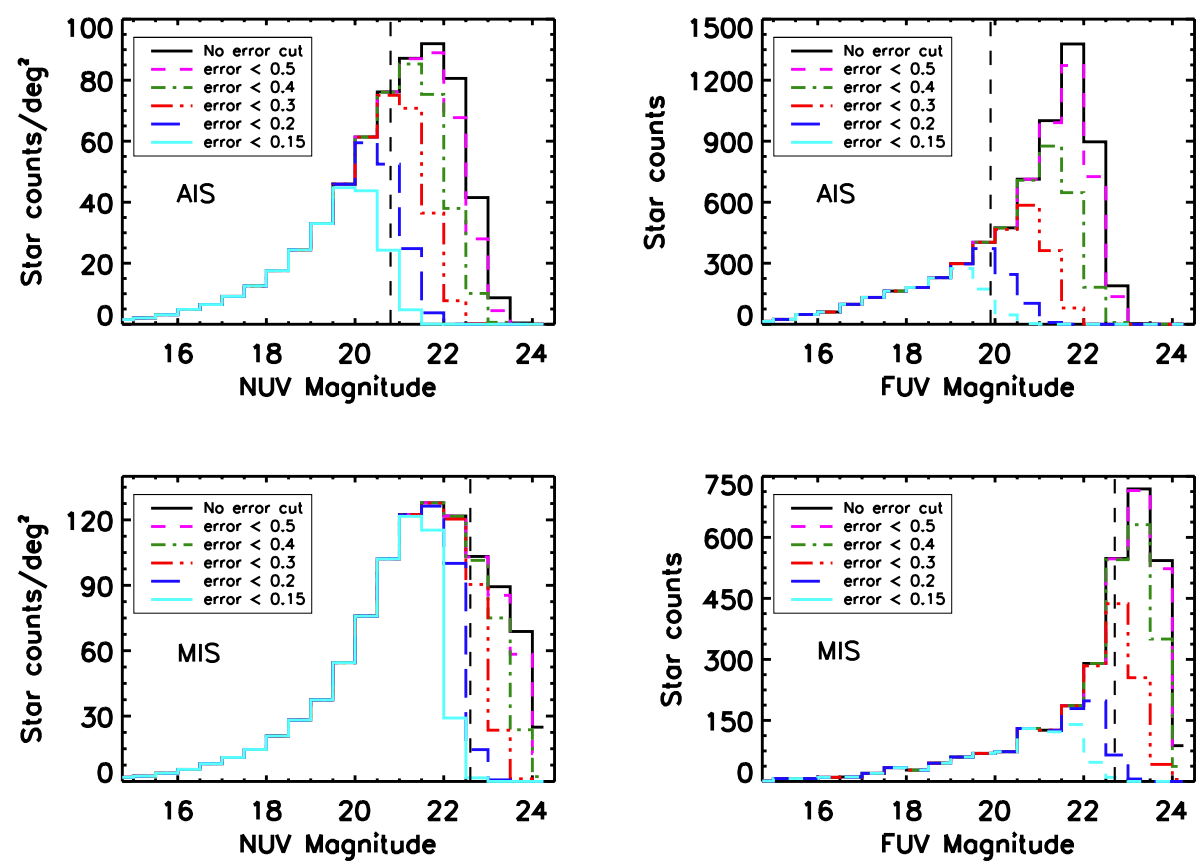

Fig. 3. Distribution of the UV-IR stars as a function of the GALEX NUV and FUV magnitudes for AIS and MIS. The stellar sources obtained after applying various magnitude error cuts are shown by different line styles in colours. The vertical dashed lines represent the respective $5 \sigma$ detection limits of the GALEX bands for typical exposure times as mentioned in Sect. 2.

For hot stars, we found that the completeness limit of GALEX NUV (AIS) is reduced by $0.5 \mathrm{mag}$ (i.e., the effective magnitude limit gets brighter). The GALEX completeness limit (AIS) of hot stars is therefore limited by the depth of WISE, and similarly by the depth of 2MASS. For cool stars, the NUV (AIS) completeness limit is the same in the GALEX catalogue alone and in the combined catalogue with the near-IR surveys.

\section{Besançon Galaxy model}

The Besançon model is a population synthesis model based on a scenario of Galactic evolution and constrained by dynamics. In the model, five populations are taken into account: thin disc, thick disc, stellar halo, bar, and bulge (Robin et al. 2012). The previous versions of the model are extensively described in Robin et al. (2003). We use the newest version of the model (version of April 2013; Robin et al. 2012), which has been upgraded to include the FUV and NUV passbands of GALEX and the upcoming UVIT/Astrosat, by applying their filter transmission curves to produce UV star counts in various Galactic directions. The model uses a set of evolutionary tracks, a star formation rate and an IMF as defined in Haywood et al. (1997), to generate different stellar populations. The colours are computed from the Basel Stellar Library (BaSeL3.1) model atmospheres (Westera et al. 2002). In this new version of the model, DA and DB type WDs are included using the evolutionary tracks and atmosphere models from Holberg \& Bergeron (2006). The luminosity functions are obtained assuming an initial-to-final-mass ratio $\left(m_{\mathrm{I}}=9.1743 m_{\mathrm{f}}-3.6147\right)$ from Kalirai $(2008)$. The distribution in age is computed assuming a lifetime on the main sequence (MS) from Eggleton et al. (1989) and a lifetime on the giant branch of $15 \%$ of the time on the MS. The repartition in DA (WD with hydrogen rich atmosphere) and DB (WD with helium rich atmosphere) is computed assuming that at $T_{\text {eff }}>40000 \mathrm{~K}$ they are all DA, and at $T_{\mathrm{eff}}<20000 \mathrm{~K}, 50 \%$ are DB with a linear variation between $20000 \mathrm{~K}$ and $50000 \mathrm{~K}$. The final luminosity function is normalised to fit Harris et al. (2006). Similarly, the BHBs are incorporated in the model by taking the evolutionary tracks from BaSTI (A Bag of Stellar Tracks and Isochrones) models (Pietrinferni et al. 2004). Ultimately, the model produces UV star counts by Monte Carlo simulations using a set of stellar atmospheric models, observational photometric errors, and extinction.

The model incorporates an extinction $\left(A_{V}\right)$ assuming an ellipsoidal distribution of diffuse absorbing matter, which follows an Einasto extinction law and is depicted by an adjustable normalisation (extinction gradient) of $0.7 \mathrm{mag} / \mathrm{kpc}$ in the $V$ band. We produced the model simulations towards various Galactic directions assuming the default extinction gradient. However, the default value of diffuse extinction $(0.7 \mathrm{mag} / \mathrm{kpc}$ in the $V$ band $)$, which may not be appropriate at low latitudes, can be adjusted by adding a few absorbing clouds with a given adhoc distance and extinction from the Schlegel et al. (1998) maps. This has been illustrated in Sect. 5.1. The ratios between UV band to visual extinction are taken to be 2.67 and 2.64 for the FUV and NUV band of GALEX, respectively, following the extinction law of Cardelli et al. (1989).

Stars in the simulated GALEX catalogue have a UV colour, FUV, and NUV magnitudes, a temperature range from $1650 \mathrm{~K}$ to $65000 \mathrm{~K}, \log g$ from -1 to 9 , all luminosity classes and a range of metallicities. In the simulations done for comparison with the GALEX observed star counts, the simulated stars are mostly MS stars $(\sim 77 \%)$ with a small contribution from giants and subgiants $(\sim 17 \%)$. The WDs are $\sim 6 \%$ of the sample and reside at the bluer end of $F U V-N U V$ colour (see Sect. 7).

\section{Comparison of the GALEX+WISE+2MASS and GALEX+SDSS stars}

Figure 4 shows the distribution of the GALEX AIS star counts (for field 5 in Table 1) as a function of NUV magnitude for the model simulation (solid line), GALEX+SDSS stars 


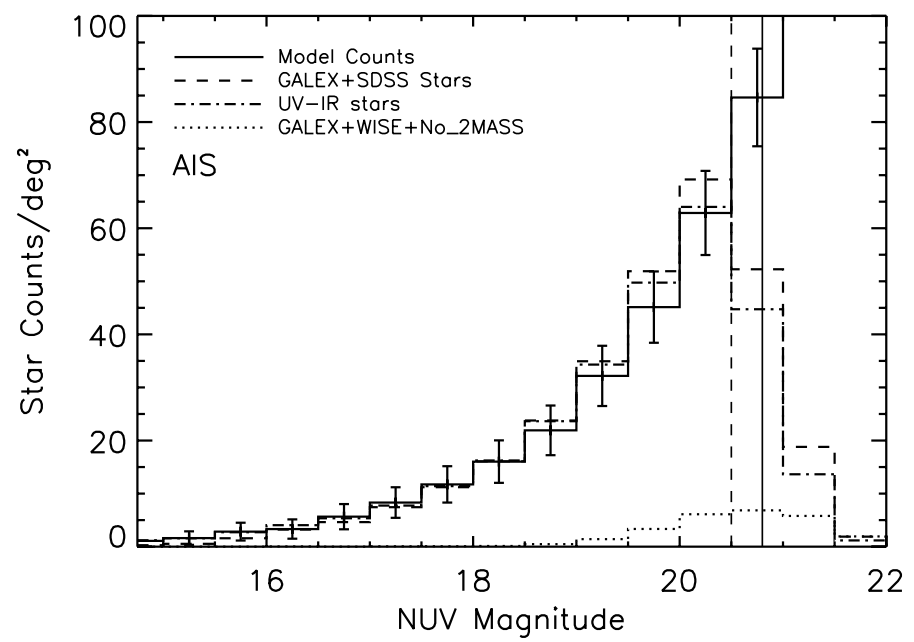

Fig. 4. Distribution of the UV-IR stars (GALEX+WISE+2MASS: dashed-dotted line), GALEX+SDSS stars (dashed line), and modelsimulated star counts (solid line) for the AIS field towards the GC, covering $69.9 \mathrm{deg}^{2}$ of the sky (field 5 in Table 1). The dotted line represents the GALEX+WISE sources with no 2MASS counterparts. The star counts are binned in 0.5 mag interval in NUV magnitude. The error bars in the model-simulated star counts are due to Poisson noise. The NUV $5 \sigma$ detection limit (NUV magnitude $=20.8$ ) is shown by a solid vertical line. The UV-IR star counts show a turn over at NUV magnitude $\sim 20.5$ (demarcated by a dashed vertical line).

(dashed line), UV-IR stars (GALEX+WISE+2MASS: dasheddotted line) and GALEX+WISE star counts with no 2MASS detection (dotted line). The error bars shown in the model star counts are due to Poisson noise. The NUV $5 \sigma$ detection limit (NUV magnitude =20.8; Morrissey et al. 2007) and the completeness limit ( 20.5 mag; see Sect. 2.5 ) for AIS are demarcated by the solid and dashed vertical lines, respectively. Stars with NUV magnitude up to the completeness limit are well detected by the GALEX, SDSS, WISE, and 2MASS surveys; a good agreement in star counts among the cross-matched surveys and the model simulations can clearly be seen in Fig. 4.

It is also evident from Fig. 4 that the GALEX+SDSS stars are slightly more than the UV-IR stars in the NUV band at the fainter magnitudes. This discrepancy could be caused by 2MASS since the time gap between the WISE and 2MASS surveys is $\sim 12$ years, high proper motion stars may have moved outside the cross-matching radii. However, stars with proper motions high enough to move by $3.0^{\prime \prime}$ in $\sim 12$ years are very rare in a survey of a few square degrees. Another possibility is that the 2MASS $J$ band, which has a $10 \sigma$ point source sensitivity limit of about $15.8 \mathrm{mag}$, does not penetrate deeply enough to provide counterparts for all WISE detections. Though GALEX+SDSS has a smaller sky area coverage and a fainter limit compared to GALEX+WISE+2MASS, both the selections yield a close match of the star counts at the brighter end. It is also possible that the GALEX+SDSS stars are still contaminated by faint galaxies and quasars. So, we preferred to use the star counts determined by the $J-W 1$ colour cut (UV-IR stars) rather than the GALEX+SDSS stars.

\section{Data and model comparison}

We modeled the stellar density distribution of the Milky Way in UV using the Besançon model of stellar population synthesis (as described in Sect. 3) for different regions of the sky. Four simulated catalogues for each of the fields chosen for our study were produced to reduce the statistical noise. Appropriate photometric errors were applied in the model to produce realistic simulations and the error information was assumed from the observed data, which is a polynomial function of the magnitude.

We can simulate the catalogues using the "small field" option, which assumes that the density does not vary across the field, or, we can simulate them using the "larger field" option with a given step in longitude and latitude, to account for the fields where the density can vary. We used the "small field" option for the small fields (e.g., area $<15 \mathrm{deg}^{2}$ ) by providing the centre $l$ and $b$ coordinates of the fields along with their coverage area. For the larger fields (e.g., area $>15 \mathrm{deg}^{2}$ ), we used the "larger field" option where we provide the range of $l$ and $b$ coordinates and a step size (e.g., from $1.0^{\circ}$ to $2.5^{\circ}$ for small to large fields, respectively) to cover the field. However, the gradients in the fields (Table 1) are small enough that considering either the centre of the field or the range of $l / b$ does not make any difference in the predicted star counts.

\subsection{Comparison of observed UV star counts with the model in various fields}

Initially, simulations were performed for four GALEX tiles (fields 1-4 in Table 1), each covering an area of $0.785 \mathrm{deg}^{2}$ at the southern intermediate Galactic latitudes. We binned the model and the UV-IR stars in 0.5 mag intervals in the NUV band, for respective tiles of AIS and MIS, in the directions of the GC and the GAC. As shown in Fig. 5, we found that the model star counts (solid line) match the UV-IR stars (solid circles) as well as the GALEX+SDSS stars (open circles) well up to the completeness limits of AIS (20.5 mag) and MIS (22.0 mag) for the regions at the southern intermediate Galactic latitudes.

In order to check the universal validity of the model, we produced simulated catalogues in various directions of the Galaxy covering a large area of the sky. Figures $6 a$ and $b$ show the comparison of the model-predicted NUV star counts (solid line) with the UV-IR stars (solid circles) as well as with the GALEX+SDSS stars (open circles) for AIS in the directions of the GC and the GAC, each covering $69.9 \mathrm{deg}^{2}$ area of the sky (fields 5-6 in Table 1). Similarly, Figs. 6c and d represent the comparison of star counts for MIS in the directions of the GC and the GAC, covering an area of $22.77 \mathrm{deg}^{2}$ and $18.85 \mathrm{deg}^{2}$, respectively (fields $7-8$ in Table 1 ). These fields are chosen at the northern intermediate Galactic latitude of the Galaxy. The error bars shown in the model-predicted star counts are due to Poisson noise. The maximum estimated asymmetric error in the observed counts is $\sim 2 \%-10 \%$, depending on the NUV magnitude bins (i.e., error increases towards the fainter magnitude bins), which is not shown in the plots. The model shows a good agreement with the observation (UV-IR stars and GALEX+SDSS stars) down to an NUV magnitude of 220.5 for AIS and 22.0 for MIS (see Figs. 5 and 6).

We also produced the model-predicted star counts for one of the passbands (NUVB4: 2505-2780 $\AA, \lambda_{\text {eff }}=2612 \AA$ ) of upcoming UVIT/Astrosat, which is shown by a dashed-dotted line. Star counts are enhanced in the UVIT NUVB4 band compared to the GALEX NUV band because NUVB4 covers a smaller wavelength range and its effective wavelength is longer than the effective wavelength of the NUV band. Most of the stars have flux peaks at longer wavelengths, such that $N U V-N U V B 4$ is positive. Since the magnitudes are normalised to the AB system, the integral of the filter does not matter while computing 


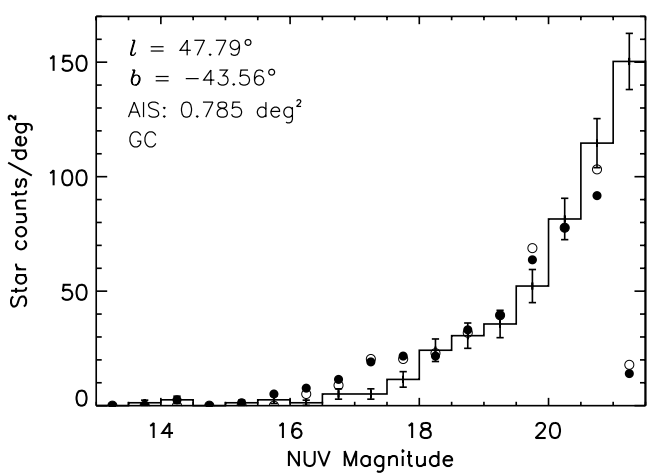

(a)

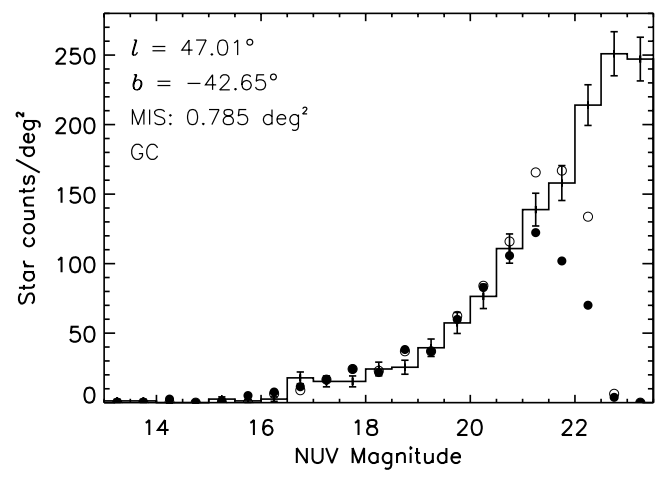

(c)

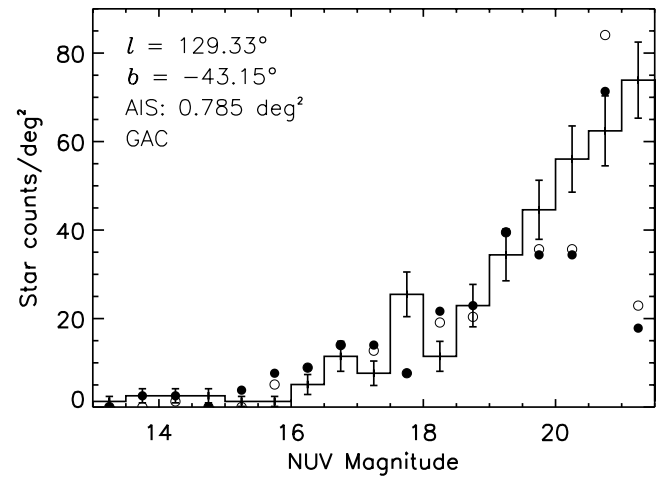

(b)

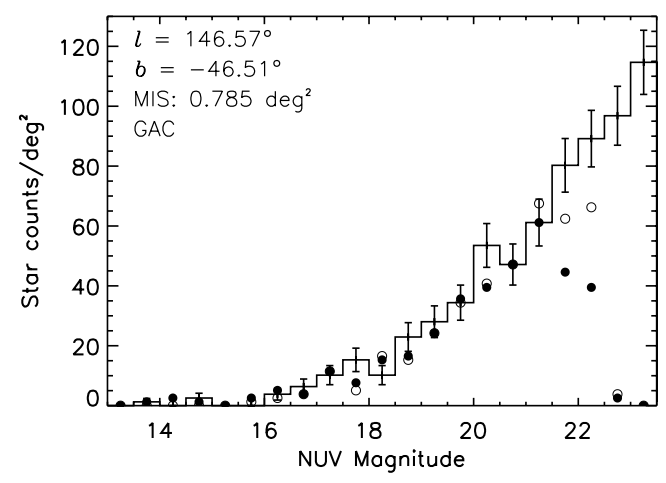

(d)

Fig. 5. Comparison of the UV-IR stars (solid circles) with model-predictions (solid line) as a function of NUV magnitudes for the regions at the southern intermediate Galactic latitudes. The open circles represent the GALEX+SDSS stars. The plots are for the fields towards the GC and the GAC for individual GALEX AIS and MIS tiles, each covering an area of $0.785 \mathrm{deg}^{2}$ (fields $1-4$ in Table 1). The error bars shown in the model star counts are due to Poisson noise, while the asymmetric errors in the observed star counts are not shown in the plot.

magnitudes, though narrower filters will demand longer exposure times to get the required magnitude.

The model-predicted star counts for the regions at the GHL, the GAR, and the GP (solid line: fields 9-12 in Table 1) match the UV-IR stars (solid circles) and the GALEX+SDSS stars (open circles) well except the region at the GLL (see Figs. 7ad). As seen in Fig. 7d, the model simulated NUV star counts (solid line) produced using the standard diffuse extinction do not match the observations beyond an NUV magnitude fainter than 18.5. This mismatch could be because of the default extinction gradient being used in the model not being sufficient at the GLL. We took the line of sight extinction $\left(A_{V}=0.1 \mathrm{mag}\right)$ for the GLL from the Schlegel et al. (1998) maps and then corrected the extinction by adding a cloud of $A_{V}=0.1 \mathrm{mag}$ at a distance of $1 \mathrm{kpc}$ (Sect. 3). The model-predicted star counts after correcting the extinction (dashed line) show a good agreement with the UV-IR stars.

In Fig. 8, we have shown the distribution in FUV magnitudes of the UV-IR stars (solid circles) and model-simulated (solid histograms) star counts for AIS and MIS (fields 5-8 in Table 1) towards the GC and the GAC. Despite the poor statistics, the model fit the observations well up to the completeness limit of the data sets (see Sect. 2.5). We have also produced the model-predicted star counts for one of the FUV passbands (BaF2: 1370-1750 $\mathrm{A}, \lambda_{\text {eff }}=1504 \AA$ ) of the forthcoming UVIT/Astrosat, which is shown by a dashed-dotted line in Fig. 8. Since the BaF2 passband range is close to the GALEX
FUV passband, the UVIT model simulated FUV star counts match the GALEX observed FUV star counts reasonably well.

Overall, the Besançon model of stellar population synthesis upgraded to the UV passbands simulates star counts, which are consistent with the observed GALEX star counts and can be used efficiently for the study of Galactic structure parameters.

\subsection{Latitude variation in star counts}

In order to study the latitude variation of UV star counts, we have chosen GALEX fields at $10^{\circ}$ Galactic latitude intervals for $l \sim 50^{\circ}$. We determined NUV star counts per square degree in each field separately for the GALEX+SDSS stars and the UV-IR stars. As shown in Fig. 9, the solid circles represent the UV-IR stars while the open circles show the GALEX+SDSS stars. The solid line represents the model generated star counts. The model errors due to Poisson noise are shown in the plot while we do not show the asymmetric errors on the UV-IR star counts which arise due to the propagation of photometric errors. The stellar density decreases from lower to higher Galactic latitudes in the case of both observed and model star counts. The UV-IR star counts with NUV magnitude brighter than 20.5 mag match model simulations at the intermediate and high Galactic latitudes. However, a slight deviation of model simulated counts from observed counts is seen at low Galactic latitudes. This could be because of the default extinction gradient used in the model which might be inappropriate at low latitudes because some clouds can be present as discussed above for Fig. 7d. 


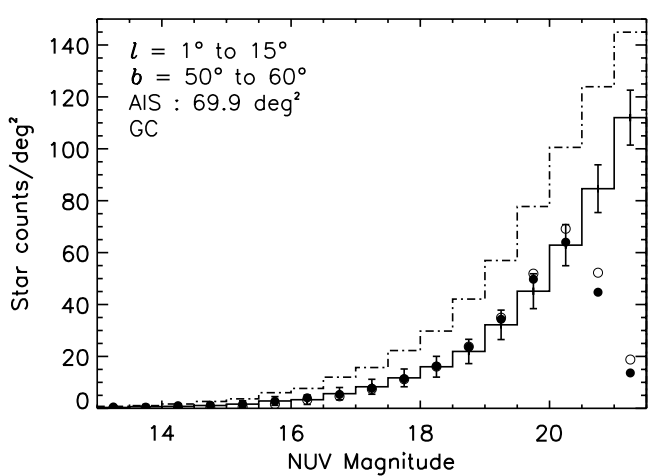

(a)

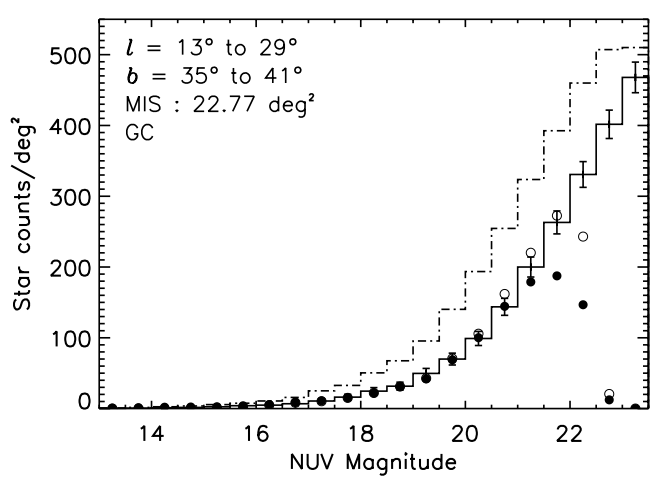

(c)

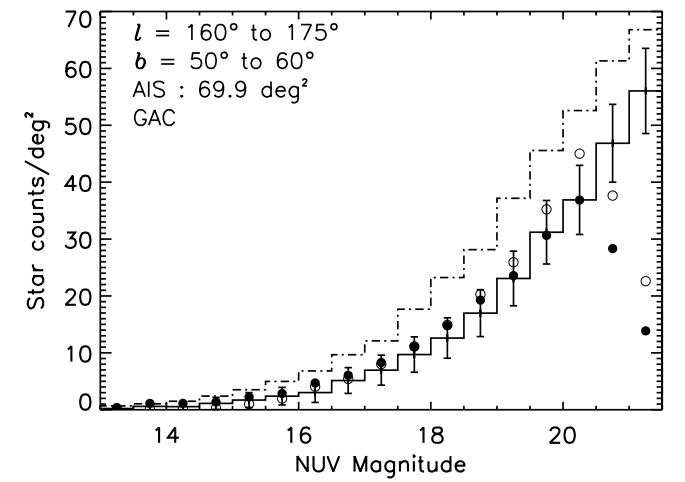

(b)

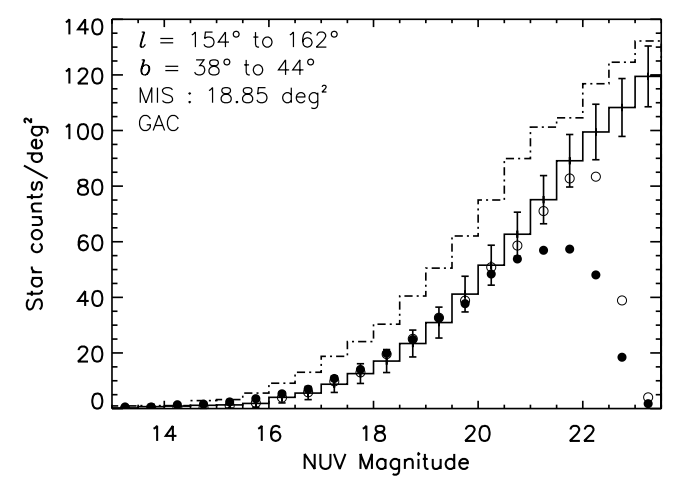

(d)

Fig. 6. Comparison of the model-predicted star counts (solid line) with the UV-IR stars (solid circles) as well as with the GALEX+SDSS stars (open circles) for the GALEX fields at the northern intermediate Galactic latitudes. The Galactic coordinate ranges, survey types, and area coverages are mentioned in each panel. The dashed-dotted line shows the model-simulated star counts for the NUVB4 band of UVIT/Astrosat (2505-2780 A, $\lambda_{\text {eff }}=2612 \AA$ ). The error bars shown in the model counts are due to Poisson noise.

\subsection{Comparison with the TRILEGAL model}

The predictions from the TRILEGAL model (Girardi et al. 2005), which is another stellar population code, have been compared with UV star counts by Bianchi et al. (2011a). It was found that the TRILEGAL-predicted NUV star counts, which show an overall good match to observations at brighter magnitudes, are better at northern high latitudes and southern low latitudes. We produced NUV star counts using the three alternative IMFs that the TRILEGAL website ${ }^{6}$ proposes. However, we see in Fig. 10 that the Besançon model produces a better fit to real star counts than TRILEGAL does in the GHL field close to the pole as well as in the GAR field at the intermediate latitudes. Here we use a WD model similar to TRILEGAL, with small differences. The initial-to-final mass relation from Kalirai (2008) is used in the Besançon model, while TRILEGAL alternatively uses Marigo \& Girardi (2007) or Weidemann (2000), the latter giving a better fit to the GALEX data (see Fig. 9 in Bianchi et al. 2011a). We also use different atmosphere models (Holberg \& Bergeron 2006), while TRILEGAL uses either Koester (2008) or TLUSTY models (Hubeny \& Lanz 1995). Bianchi et al. (2011a) pointed out that the difference between these two models is not larger than 0.05 mag in $F U V-N U V$ colour for most of the WDs. Finally, TRILEGAL does not consider DB WDs because it includes only WDs hotter than $18000 \mathrm{~K}$, although we have taken them into account. However, the difference between TRILEGAL and the Besançon model predictions is mainly because of the

\footnotetext{
6 http://stev.oapd.inaf.it/cgi-bin/trilegal
}

more detailed account for the settling of the disc with age in the Besançon Galaxy model (the dynamical constraint which is used, forces the sub-components of the thin disc to follow a tied age/vertical scale height relation in agreement with the observed age/velocity dispersion relation).

\section{Distribution of the stars}

We find that the model reproduces the observed UV star counts as selected from the GALEX data. The star counts are dominated by MS stars, WDs, and BHBs. The vertical distribution of different stellar populations depends on their structural parameters. In Fig. 11a, we show the contribution of the thin disc (dotted line), thick disc (dashed line), halo (dashed-dotted line), and sum of the three populations (solid line) predicted by the model for an AIS field towards the GC at the intermediate Galactic latitude. The relatively bright stars are dominated by the thin disc at NUV magnitudes brighter than 18.5 whereas thick disc and halo stars become significant at NUV magnitudes fainter than $\sim 18.5$ and $\sim 19.5$, respectively. This is very similar to the comparison made by Bianchi et al. (2011a) for hot star candidates. Considering the stars with NUV magnitudes brighter than 20.5, we found that thick disc stars are the most dominant population and $\sim 54 \%-\sim 60 \%$ of the total population (depending on the Galactic direction).

We have shown the vertical distribution of the model simulated stars in Fig. 11b. It is evident that thin disc star counts (dotted line) dominate up to a distance of $1.5 \mathrm{kpc}$ over the 


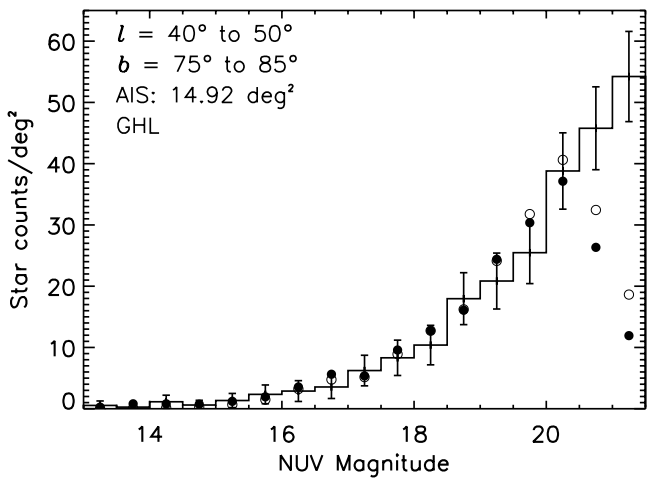

(a)

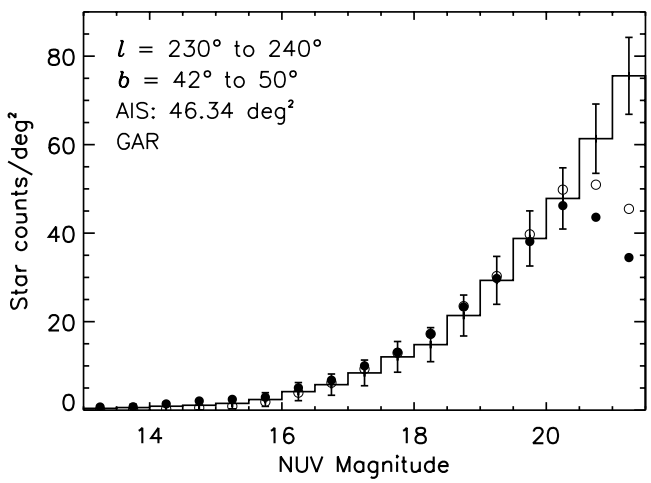

(c)

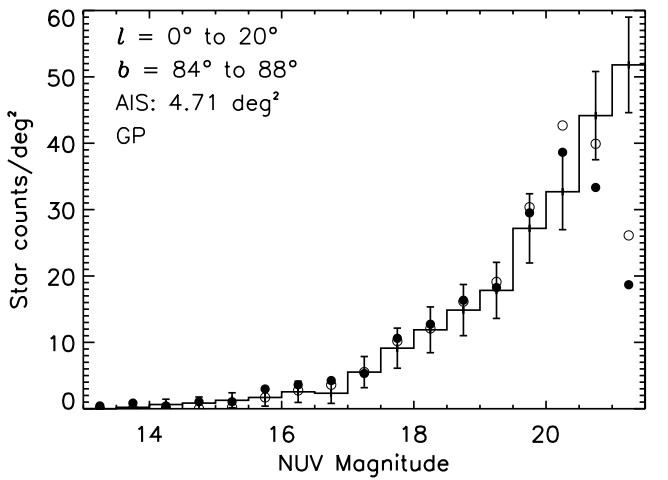

(b)

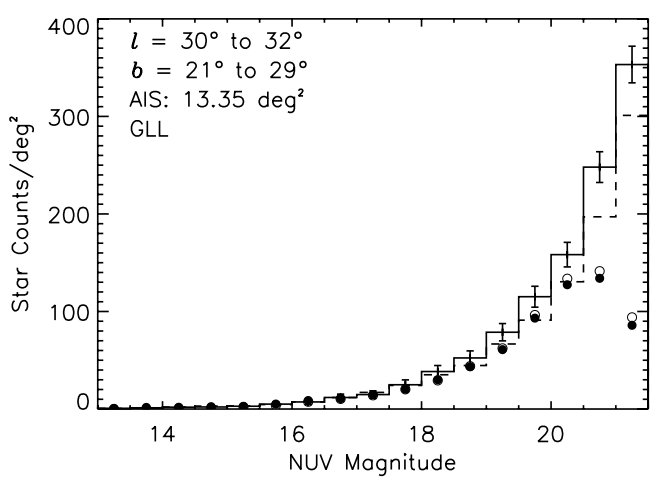

(d)

Fig. 7. Comparison of model-predictions (solid line) with the UV-IR stars (solid circles) and GALEX+SDSS stars (open circles) as a function of the GALEX NUV magnitude for the regions at the GHL (7a), the GP (7b), the GAR (7c), and the GLL (7d). In plot 7d, the solid line represents the model NUV star counts produced assuming the standard diffuse extinction (as in other fields) whereas the dashed line is the same after correcting the extinction using the value from the Schlegel et al. (1998) maps (see Sect. 5). The model error bars shown in the plots are due to Poisson noise.

Galactic plane whereas thick disc star counts (dashed line) dominate at distances between 1.5 and $4.0 \mathrm{kpc}$ beyond which halo stars (dashed-dotted line) dominate the total stellar population. A similar trend has been observed by both Du et al. (2003) for BATC (Beijing-Arizona-Taiwan-Connecticut) multicolour photometric survey star counts and Phleps et al. (2000) for CADIS (Calar Alto Deep Imaging Survey) deep star counts for regions at intermediate Galactic latitudes.

\section{Blue hot stars}

The $F U V-N U V$ colour is an important indicator of the spectral type of the stars. Particularly, UV colour can be used to identify hot BHBs and WDs (Kinman et al. 2007; Bianchi et al. 2011a), which emit most of their light in UV because of their high temperatures. The BHBs are comparatively more luminous in UV than the other population II stars. Similarly, the WDs, which are the end product of the stellar evolution of the intermediate and low mass stars, provide important information about the Galactic disc star formation history. Comparing the observed $F U V-N U V$ colour of stars with the model, we were able to separate out the halo BHBs and disc WDs from the whole sample of stars.

Figure 12 shows the comparison of GALEX FUV - NUV colours for the UV-IR stars (solid circles) and model simulated star counts (solid-lined histogram) for the AIS fields towards the GC and the GAC. We have considered stars with NUV magnitude $<20.5$ and FUV magnitude $<20.0$ for the GALEX AIS survey. The $F U V-N U V$ colours of WD (dotted line) and BHB (long-dashed line) populations are also shown along with the UVIT FUV - NUV (BaF2 - NUVB4) colour (dashed line) in the plot. Looking at the $F U V-N U V$ model predictions, the stars can be classified into two groups: one group of stars with $F U V-N U V>2.5$ are the red cool stars, and the other group of stars with $F U V-N U V<2.5$ are blue hot stars. The blue stars exhibit a bimodal distribution indicating the existence of two populations; the peak at $F U V-N U V \sim-0.5$ are the hot WDs of the disc, and the peak at $F U V-N U V \sim 2.0$ are BHBs of the Galactic halo. In the Besançon model, the temperature range of WDs is from $10000 \mathrm{~K}$ to $27000 \mathrm{~K}$ and that of $\mathrm{BHBs}$ is from $5000 \mathrm{~K}$ to $20000 \mathrm{~K}$. Hotter stars with temperature greater than $27000 \mathrm{~K}$ are rarely found in significant numbers in the data considered here.

The colour distributions in Fig. 12 towards both the GC and the GAC show some differences between the model and observations. Especially, we notice that the very blue peak at $F U V-$ $N U V<0$, due to hot WDs, is too high in the model. Moreover, there is a lack of stars in the GC field at $0<F U V-N U V<1.5$. In the colour range where the BHBs dominate, the number of predicted stars is well in agreement with the observations in both fields, indicating that the halo BHB density is well simulated. There is a dearth of model-simulated stars in the colour range, $2<F U V-N U V<3.5$, which is not understood yet and will be investigated in a further study. At $F U V-N U V>4$, the model lacks stars but this is the case more towards the GAC than towards the GC. This colour domain is mostly dominated by thick disc MS stars. We guess that it is because of the scale length which will be investigated in a forthcoming paper. 

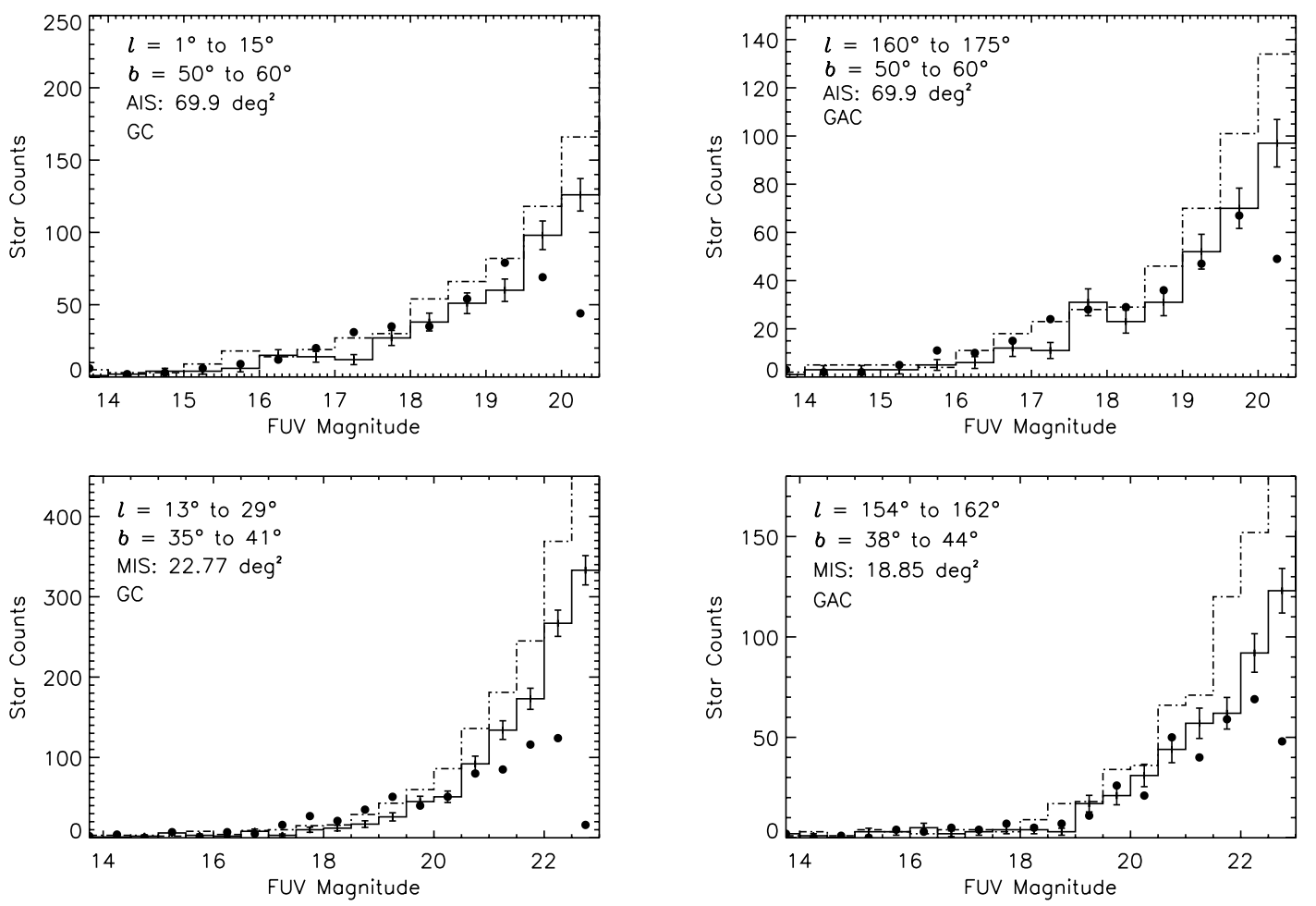

Fig. 8. Distribution in FUV magnitudes of the UV-IR stars (solid circles) and model-predicted (solid line) star counts for the AIS and MIS fields towards the GC and the GAC (fields 5-8 in Table 1). The dashed-dotted line represents FUV star counts for the BaF2 band of UVIT/Astrosat $\left(1350-1750 \AA, \lambda_{\text {eff }}=1504 \AA\right)$. The FUV magnitudes are binned in intervals of 0.5 . The model error bars shown in the plots are due to Poisson noise.

Both photometry and spectroscopy can be used to identify WDs and BHBs. Several large area sky surveys such as 2MASS, SDSS, and GALEX have been used to distinguish them with appropriate colour selections and it is worth mentioning a few of the works. Kleinman et al. (2013) produced the latest catalogue of spectroscopically confirmed DA- and DB-type WDs from SDSS Data Release 7. Using the data from GALEX FUV and NUV imaging, Bianchi et al. (2011b) presented a catalogue of hot star candidates, particularly WDs. Similarly, the first selection of BHBs from SDSS colours was made by Yanny et al. (2000) and then followed by many others (Sirko et al. 2004; Bell et al. 2010; Deason et al. 2011; Vickers et al. 2012). We have identified WD and BHB candidates using suitable GALEX $F U V-N U V$ colours. It was found from the model $F U V-N U V$ colour (Fig. 11) that BHB and WD star candidates occupy the colour range, $1.5<F U V-N U V<2.5$ and $F U V-N U V<0.5$, respectively. In the mentioned colour range, we obtain a clean sample of WD candidates, whereas in the sample of BHB candidates, a contamination of non-BHB candidates, such as WDs and MS stars, constitute about 7\%. These colour ranges have been used for the separation of WD and BHB candidates from other populations in the observed sample.

In order to substantiate our identification of the WD and BHB star candidates using GALEX $F U V-N U V$ colour, we compared them with their known 2MASS colours. The $E(B-V)$ values for the stars were measured from Schlegel et al. (1998) and converted to NUV, $J$ and $H$ extinction using Cardelli et al. (1989) extinction law: $A(N U V)=8.90 E(B-V), A(J)=$ $0.874 E(B-V)$, and $A(H)=0.589 E(B-V)$. Figure 13a shows the $(J-K)_{\mathrm{o}}$ versus $(N U V-J)_{\mathrm{o}}$ colour-colour diagram for the BHB candidates. The sources at different latitude intervals are represented by different symbols. The dashed parallelogram encloses

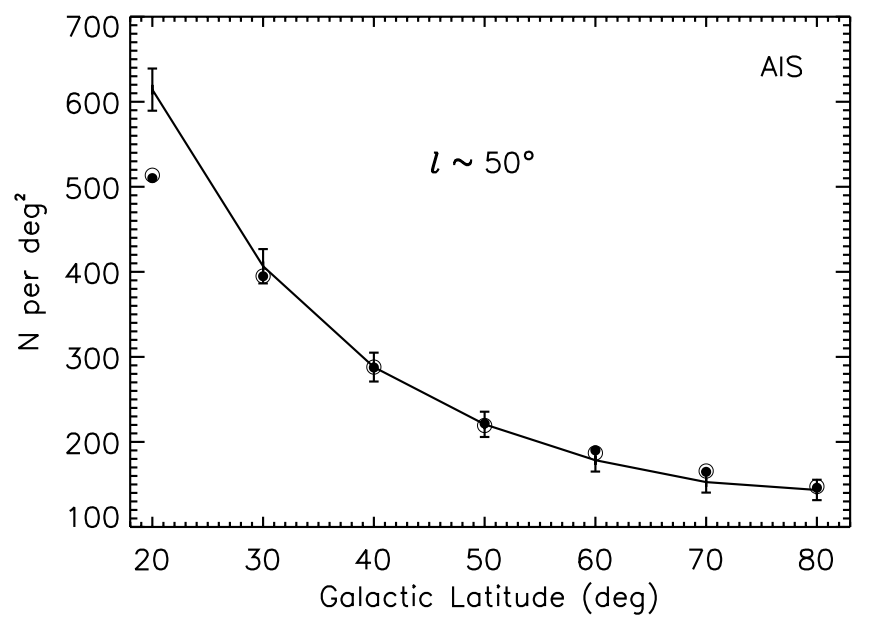

Fig. 9. Latitude variation of NUV star counts $\left(\right.$ per $\left.\mathrm{deg}^{2}\right)$ for both the observation and model simulation at $l \sim 50^{\circ}$. The UV-IR stars, GALEX+SDSS stars, and model-predicted star counts are represented by solid circles, open circles, and solid line, respectively. The UV-IR stars and the GALEX+SDSS stars shown in the plot are for NUV magnitude errors $<0.2$. The error bars displayed in the model star counts are due to Poisson noise. The asymmetric errors in the UV-IR star counts which arise because of the propagation of photometric errors are not shown.

the area used by Kinman et al. (2007), which contains $66 \%$ of the BHB candidates selected on the basis of $F U V-N U V$ colour. Similarly, Fig. 13b shows the $H-K$ versus $J-H$ colour-colour diagram for the WD candidates. The dashed rectangle encloses the area in the colour-colour diagram chosen from Hoard et al. (2007) that contains a majority of the WD candidates of our 

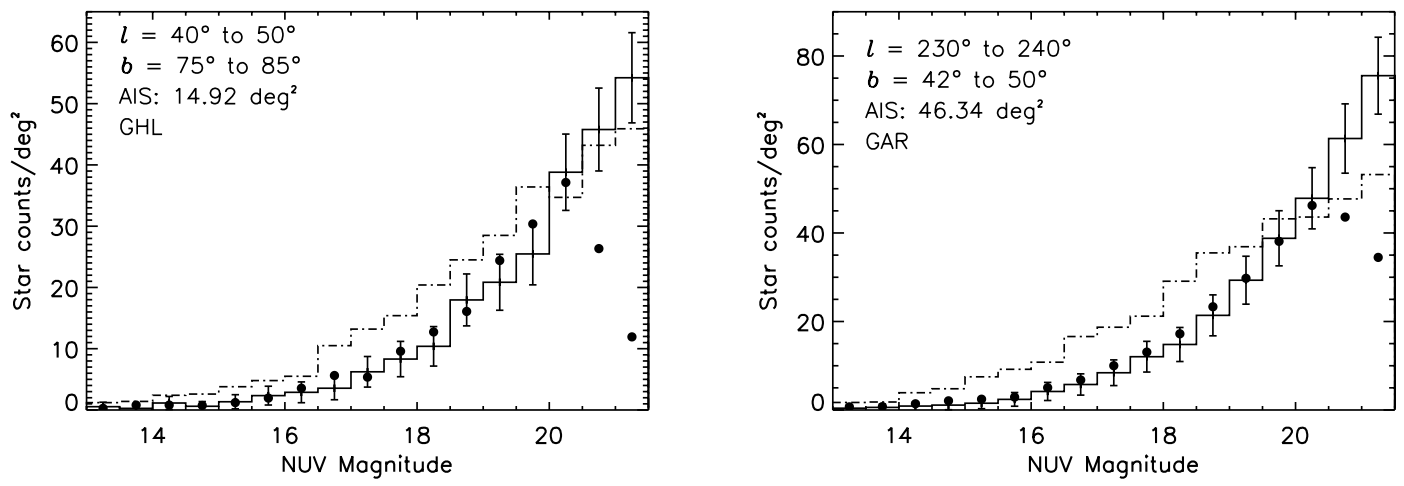

Fig. 10. Comparison of NUV star counts predicted by the Besançon (solid line) and TRILEGAL (dashed-dotted line) models of stellar population synthesis for the fields towards the GHL and the GAR. The solid circles represent the UV-IR stars. The Besançon model error bars shown in the plots are due to Poisson noise.
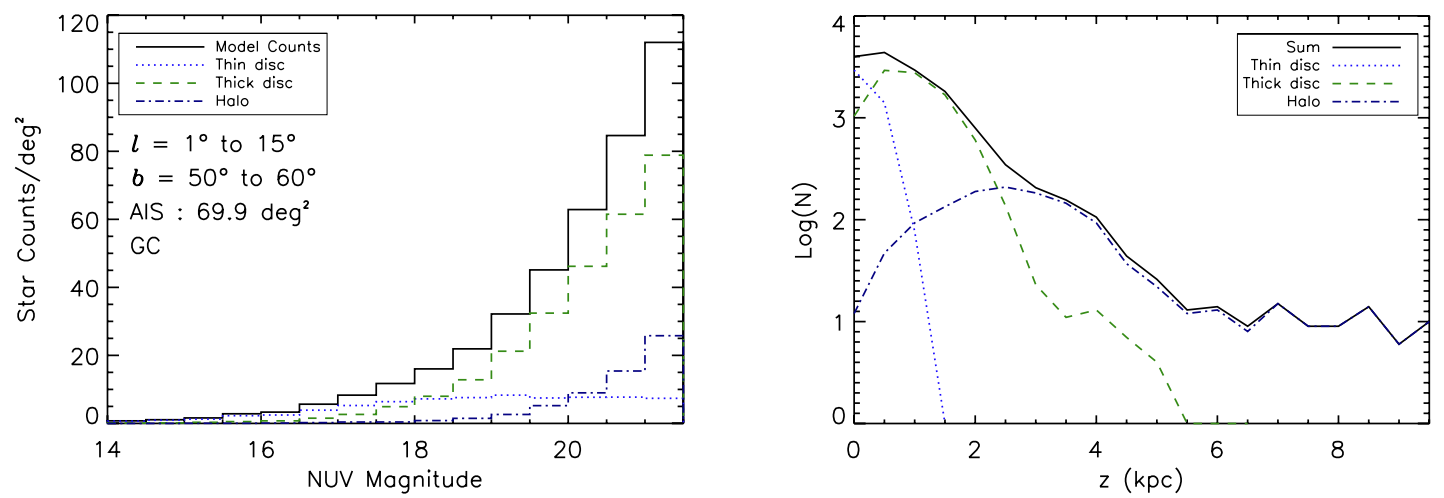

Fig. 11. Left panel: distribution of various stellar populations produced by the Besançon model of stellar population synthesis in the direction of GC. Different lines are explained in the legend. Right panel: vertical distribution of the stellar populations in the same direction as the left panel.
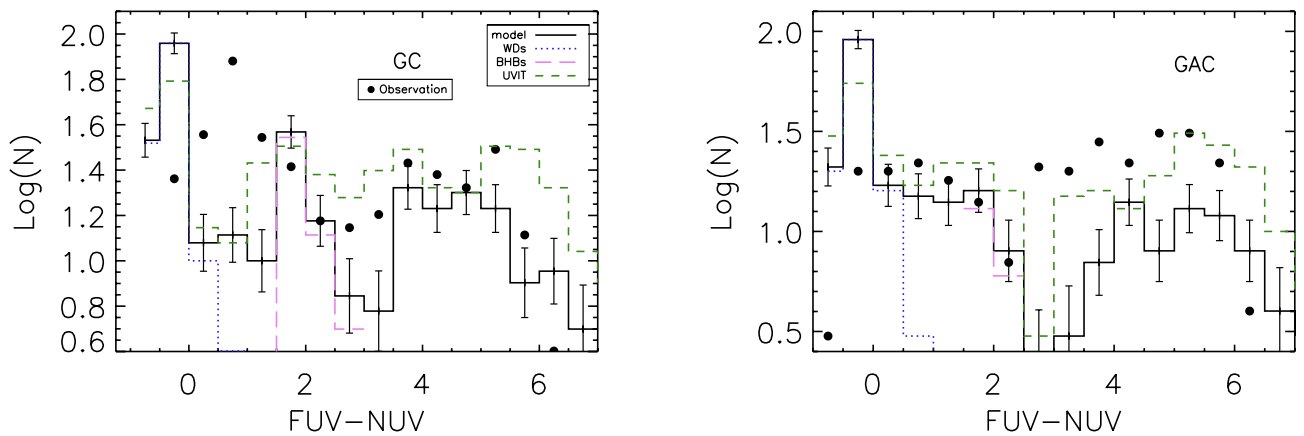

Fig. 12. Comparison of $F U V-N U V$ colour between the model-predicted star counts (solid line) and the UV-IR stars (solid circles) for the AIS fields towards the GC and the GAC (fields 5-6 in Table 1). The UV colours shown are for the stars with $F U V<20.0$ mag, $N U V<20.5$ mag, and the photometric errors $<0.2$ (in both FUV and NUV bands). FUV $-N U V$ colours of the model-predicted WDs and BHBs are shown by a dotted line and a long-dashed line, respectively. The UVIT (BaF2 - NUVB4) colour coverage is indicated by a dashed line.

sample. The location of our selected WD and BHB star candidates in the respective 2MASS colour window indicates that the $F U V-N U V$ colour can also be used as a potential tool in identifying WD and BHB candidates. This is a preliminary investigation and we will use this in our future work of an all sky study of these sources using the GALEX data.

\section{Conclusions}

The Besançon model of stellar population synthesis has been previously checked at many different wavelengths from visible ( $U$ band) to mid-IR $(12 \mu \mathrm{m})$. The model produces accurate star counts up to magnitude $\sim 22$ in the visible or 18 in the $K$ band. However, the stars that dominate the counts in the UV were not previously checked vis-a-vis model predictions. The availability of the GALEX data gives us the opportunity to check model predictions for high temperature blue stars, especially BHBs from the halo and WDs from the disc. We have shown that the model performs very well for these types of stars as it does for other types. The model provides a good check that the population synthesis scheme gives predictions which are consistent with each other at all wavelengths. To do so, we make use of Holberg \& Bergeron (2006) models which provide good stellar atmospheres and cooling tracks for WDs. However, the ratio between DAand DB-type WDs has to be investigated more deeply.

We have generally considered a simple dust distribution while limiting the comparisons to $|b|>20^{\circ}$. In the future, we will compare the model at lower latitudes, in particular for the 


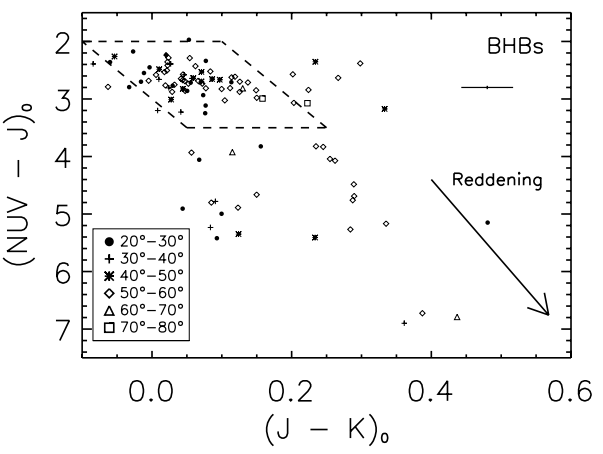

(a)

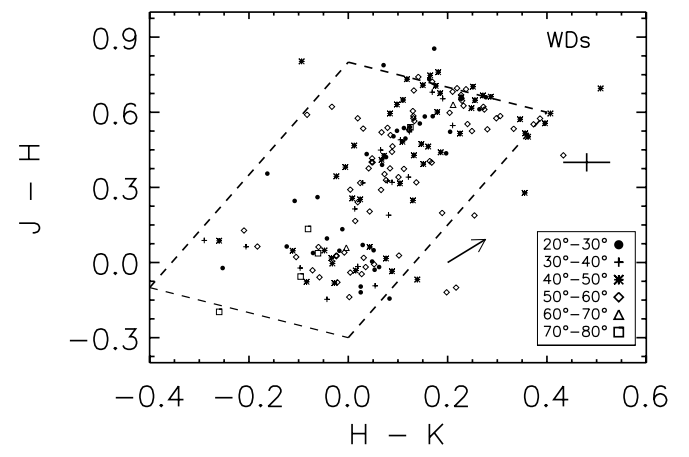

(b)

Fig. 13. a) $(J-K)_{0}$ versus $(N U V-J)_{0}$ colour-colour diagram for the BHB candidates of the combined AIS fields given in Table 1 . The parallelogram encloses the area occupied by the BHB samples of Kinman et al. (2007). The sources at various latitudes are represented by different symbols. b) $H-K$ versus $J-H$ colour-colour diagram for the WD candidates of the combined AIS fields in Table 1 . The dashed line rectangle encloses the area used from Hoard et al. (2007). A reddening vector (the arrow) of $A_{V}=1$ mag and the mean error bars of the colours are displayed in both the diagrams.

sake of analysis of the spiral structure, assuming the 3D extinction map from Marshall et al. (2006).

We also compared predictions in the UV bands from the TRILEGAL model with our model and found that the predictions of the Besançon model are in better agreement with the observation than the TRILEGAL model as shown in Fig. 10. However, in the faintest NUV magnitude bins, TRILEGAL seems to be better in the GAR field. It will be something to look at carefully in the future using the all sky observations of GALEX and WISE, and we aim to present a detailed comparison between observations and the model.

We plan to complete the analysis by comparing model predictions with a variety of models of WDs, varying the tracks, and investigating whether it could be possible to constrain the star formation history of the disc from the WDs distribution. Moreover, an analysis of the thick disc WD luminosity function could also be interesting for constraining the formation history of this old population, but it would require complementary kinematical data. We have seen that BHBs are a major component of GALEX stars. An analysis of this component could lead to constraints on the shape of the halo, once the contamination by extra-galactic objects is eliminated.

The final model can be safely used to predict star counts of various types in UV wavelengths at the level of a few percent in many Galactic directions; the model produces star counts that match well down to $F U V \sim 20.0, N U V \sim 20.5$ for AIS, and $F U V \sim 22.5, N U V \sim 22.0$ for MIS. However, for the hot WDs, there is a mismatch of UV colours between the model and observation. A more detailed study is planned to explain the discrepancies by changing the WD luminosity function and the scale lengths alternatively. A study is also underway on to better constrain the thick disc shape from large surveys in the visible and near-IR (Robin et al., in prep.). We plan to further investigate the UV star counts with this revised model and the GALEX survey in the near future.

We developed the Besançon model to predict star counts in the UV passbands of the forthcoming UVIT telescope to be flown onboard Astrosat. We compared the model-predicted star counts at two of the UVIT filters with that of the GALEX observed star counts because of the similar wavelength coverage of both the instruments. The UVIT-predicted star counts are sensitively different from the GALEX observed star counts because of the differences in effective wavelengths. UVIT star counts will be very useful to separate out different stellar populations since they have several UV colours and better angular resolution compared to GALEX, which in turn will help us to estimate the structural parameters of the Galaxy with better precision.

Acknowledgements. The authors thank the anonymous referee for useful comments and suggestions that improved the content of the paper. GALEX (Galaxy Evolution Explorer) is a NASA small explorer launched in 2003 April. We gratefully acknowledge NASA's support for construction, operation, and science analysis for the GALEX mission, developed in cooperation with the Centre National d'Etudes Spatiales of France and the Korean Ministry of Science and Technology. This work has made use of the data products from the Widefield Infrared Survey Explorer (WISE), Two Micron All Sky Survey (2MASS) and Sloan Digital Sky Survey (SDSS). We also thank the UVIT/Astrosat team for providing the UVIT filter response curves. Simulations were executed on computers from the Utinam Institute of the Université de Franche-Comté, supported by the Région de Franche-Comté and Institut des Sciences de l'Univers (INSU). We acknowledge the support of the French "Agence Nationale de la Recherche" under contract ANR-2010-BLAN-0508-01OTP. Many thanks to Bernard Debray, who is responsible for providing the web interface for the Besançon Galaxy model. This work was fully or partially supported by the Gaia Research for European Astronomy Training (GREAT-ITN) Marie Curie network, funded through the European Union Seventh Framework Programme (FP7/2007-2013) under grant agreement No. 264895. J.J.V. is a fellow at the International Max Planck Research School for Astronomy and Cosmic Physics at the University of Heidelberg.

\section{References}

Aihara, H., Allende Prieto, C., An, D., et al. 2011, ApJS, 193, 29

Bahcall, J. N. 1986, ARA\&A, 24, 577

Bahcall, J. N., \& Soneira, R. M. 1980, ApJ, 238, 17

Bell, E., Xue, X., \& Rix, H.-W. 2010, AJ, 140, 1850

Bianchi, L. 2009, Ap\&SS, 320, 11

Bianchi, L., Rodriguez-Merino, L., Viton, M., et al. 2007, ApJS, 173, 659

Bianchi, L., Efremova, B., Herald, J., et al. 2011a, MNRAS, 411, 2770

Bianchi, L., Herald, J., Efremova, B., et al. 2011b, Ap\&SS, 335, 161

Bianchi, L., Conti, A., \& Shiao, B. 2014, J. Adv. Space Res., 53, 900

Brosch, N. 1991, MNRAS, 250, 780

Budavári, T., Heinin, S., Szalay, A. S., et al. 2009, ApJ, 694, 1281

Cardelli, J. A., Clayton, G. C., \& Mathis, J. S. 1989, ApJ, 345, 245

Cohen, M., Saseen, T. P., \& Bowyer, S. 1994, ApJ, 427, 848

Deason, A., Belokurov, V., Evans, N., et al. 2011, MNRAS, 416, 2903

Du, C., Zhou, X., Ma, J., et al. 2003, A\&A, 407, 541

Eggleton, P. P., Fitchett, M. J., Tout, C. A., et al. 1989, ApJ, 347, 998

Freeman, K. C. 1987, ARA\&A, 25, 603

Gilmore, G., \& Reid, N. 1983, MNRAS, 202, 1025

Gilmore, G., Wyse, R. F. G., \& Kuijken, K. 1988, ARA\&A, 27, 555

Girardi, L., Groenewegen, M. A. T., Htziminaoglou, E., \& da Costa, L. 2005, A\&A, 436, 895

Guillout, P., Haywood, M., Motch, C., \& Robin, A. C. 1996, A\&A, 316, 89

Harris, H. C., Munn, J. A., \& Kilic, M. 2006, AJ, 131, 571 
Haywood, M., Robin, A. C., \& Crézé, M. 1997, A\&A, 320, 440 Helmi, A. 2008, A\&ARv, 15, 145

Hoard, D. W., Wachter, S., \& Sturch, L. K. 2007, AJ, 134, 26

Holberg, J. B., \& Bergeron, P. 2006, AJ, 132, 1221

Hubeny, I., \& Lanz, T. 1995, ApJ, 439, 875

Ivezić, Ż., Beers, T. C., \& Jurić, M. 2012, ARA\&A, 50, 251

Jurić, M., Ivezić, Ž., Brooks, A., et al. 2008, ApJ, 673, 864

Kalirai, J. S., Hansen, B. M. S., \& Kelson, D. D. 2008, ApJ, 676, 594

Kapteyn, J. C. 1922, ApJ, 55, 302

Kinman, T. D., Salim, S., \& Clewey, L. 2007, ApJ, 662, L111

Kleinman, S. J., Kepler, S. O., \& Koester, D. 2013, ApJS, 204, 5

Koester, D. 2008, Mem. Soc. Astron. It., 75, 282

Kumar, A., Ghosh, S. K., Hutchings, J., et al. 2012a, Space Telescopes and Instrumentation 2012: Ultraviolet to Gamma Ray, Proc. SPIE, 8443

Kumar, A., Ghosh, S. K., Kamath, P. U., et al. 2012b, Space Telescopes and Instrumentation 2012: Ultraviolet to Gamma Ray, Proc. SPIE, 8443 Majewski, S. R. 1993, ARA\&A, 31, 575

Marshall, D. J., Robin, A. C., Reylé, C., et al. 2006, A\&A, 453, 635

Marigo, P., \& Girardi L. 2007, A\&A, 469, 239

Martin, D. C., Fanson, J., Schiminovich, D., et al. 2005, ApJ, 619, L

Morrissey, P., Schiminivoch, D., Barlow, T. A., et al. 2005, ApJ, 619, L7

Morrissey, P., Conrow, T., Barlow, T. A., et al. 2007, ApJS, 173, 682
Phleps, S., Meisenheimer, K., Fuchs, B., \& Wolf, C. 2000, A\&A, 356, 108 Ohlendorf, H., Preibisch, T., \& Gaczkowski, B. 2013, A\&A, 552, A14 Pietrinferni, A., Cassisi, S., Salaris, M., \& Castelli, F. 2004, ApJ, 612, 168

Postma, J., Hutchings, J. B., \& Leahy, D. 2011, PASP, 123, 833

Richards, G. T., Fan, X., Newberg, H. J., et al. 2002, AJ, 123, 2945

Robin, A. C., \& Crézé, M. 1986, A\&A, 157, 71

Robin, A. C., Reylé, C., Derriére, S., \& Picaud, S. 2003, A\&A, 409, 523

Robin, A. C., Marshall, D. J., Schultheis, M., \& Reylé, C. 2012, A\&A, 538, A106

Schlegel, D.J., Finkbeiner, D.P., \& Davis, M. 1998, ApJ, 500, 525

Seibert, M., Budavári, T., Rhee, J., et al. 2005, ApJ, 619, L23

Sirko, E., Goodman, J., Knapp, G. R., et al. 2004, AJ, 134, 2236

Skrutskie, M. F., Cutri, R. M., Stiening, R., et al. 2006, AJ, 131, 1163

Todmal, S. S., Ghosh, S. K., Ojha, D. K., \& Robin, A. C. 2010, Interstellar Matter and Star Formation: A Multiwavelength Perspective, ed. by D. K. Ojha, ASI Conf. Ser., 1, 245

Vickers, J. J., Grebel, E. K., \& Huxor, A. P. 2012, AJ, 143, 86

Weidemann, V. 2000, A\&A, 363, 647

Westera, P., Lejeune, T., Buser, R., et al. 2002, A\&A, 381, 524

Wright, E. L., Eisenhardt, P. R. M., Mainzer, A. K., et al. 2010, AJ, 140, 1868

Xu, C. K., Donas, J., Arnouts, S., et al. 2005, ApJ, 619, L11

Yanny, B., Newberg, H. J., Kent, S., et al. 2000, ApJS, 540, 825 